\title{
New Analyses of Duopoly Game with Output Lower Limiters
}

\author{
Zhaohan Sheng, ${ }^{1}$ Jianguo Du, ${ }^{2,3}$ Qiang Mei, ${ }^{2}$ and Tingwen Huang ${ }^{4}$ \\ ${ }^{1}$ School of Management Science and Engineering, Nanjing University, Nanjing 210093, China \\ ${ }^{2}$ School of Management, Jiangsu University, Zhenjiang 212013, China \\ ${ }^{3}$ Computational Experiment Center for Social Science, Jiangsu University, Zhenjiang 212013, China \\ ${ }^{4}$ Texas A\&M University at Qatar, P.O. Box 23874, Doha, Qatar
}

Correspondence should be addressed to Jianguo Du; iconiphotels@gmail.com

Received 23 October 2012; Accepted 30 December 2012

Academic Editor: Chuandong Li

Copyright (C) 2013 Zhaohan Sheng et al. This is an open access article distributed under the Creative Commons Attribution License, which permits unrestricted use, distribution, and reproduction in any medium, provided the original work is properly cited.

In the real business world, player sometimes would offer a limiter to their output due to capacity constraints, financial constraints, or cautious response to uncertainty in the world. In this paper, we modify a duopoly game with bounded rationality by imposing lower limiters on output. Within our model, we analyze how lower limiters have an effect on dynamics of output and give proof in theory why adding lower limiters can suppress chaos. We also explore the numbers of the equilibrium points and the distribution of conditioned equilibrium points. Stable region of the conditioned equilibrium is discussed. Numerical experiments show that the output evolution system having lower limiters becomes more robust than without them, and chaos disappears if the lower limiters are big enough. The local or global stability of the conditional equilibrium points provides a theoretical basis for the limiter control method of chaos in economic systems.

\section{Introduction}

Since the French economist Cournot [1] introduced the first well-known model which gives a mathematical description of competition in a duopolistic market, there are many research works based on it (see [2-9]). The Cournot duopoly model represents an economy consisting of two quantity-setting firms producing the same good, or homogeneous goods, and each firm chooses its production in order to maximize its profits (see [2]). Rand [10] may be the first man who suggested that the Cournot adjustment process may also fail to converge to a Nash equilibrium and may also exhibit cyclical and even chaotic dynamics. Puu [11, 12] suggested a case of Cournot duopoly with two or there players, and with a unitary elasticity demand function and constant marginal costs, and showed that these systems also lead to complex dynamics, including period doubling bifurcations and chaos. Kopel [13] investigated microeconomic foundations of Cournot duopoly games and demonstrated that cost functions incorporating an interfirm externality lead to a system of coupled logistic equations. Besides extending Puu's work to $n$ competitors (see [6]), Ahmed et al. [7] contributed to develop dynamic Cournot game characterized by players with complete rationality into one with bounded rationality. After them, scholars studied Cournot game with bounded rationality, considering influence of different demand functions and different cost functions (linear and nonlinear) (see $[2,3,9]$ ). Recently, some scholars (see $[4,5,14-20]$ ) introduced heterogeneous players into Cournot game with bounded rationality. Aims of all the modifications previously mentioned or not are to make Cournot game model become economically more justified in the world.

In the real business world, it is commonly observed that competitive firms would limit their production for steadiness or economies of scale. Huang [21] found that cautious responses to fluctuating prices by firms (through limiting the growth rate of its output) may result in a higher longrun average profit for a simple cobweb. He and Westerhoff [22] showed that imposition of a price limiters can eliminate homoclinic bifurcations between bull and bear markets and hence reduce market price volatility. It is worthy to be noticed that work of $[21,22]$ is based on one-dimension economic system. In incomplete competition, the number of players is 
not less than 2, so the problem may become more intricate (see [23]).

This paper aims at the new output duopoly game by imposing lower limiters on output and focuses on the impact of limiters on dynamics and unraveling stabilizing mechanism of limiter method to reduce fluctuation.

As He and Westerhoff [22] say, output limiters as applied model are identical to a recently developed chaos control method, the limiter method, which has been analytically and numerically explored by [22-26]. However, the existent documents do not discuss the impact of limiter on equilibrium of economic system. Unfortunately, there exists no theoretical result to assure the fact that chaos can be suppressed by adding simple limiter. The results in this paper may partly answer this puzzle in a special case.

The remainder of this paper is organized as follows. Section 2 introduces an output duopoly game with bounded rationality and examines the dynamics of the model. Section 3 turns to a discussion of the duopoly game having output lower limiters and the impact of output lower limiters on dynamics. The final section concludes the paper.

\section{The Output Duopoly Game without Output Limiter}

The output game we introduce here is based on the assumption that the two firms (players) do not have a complete knowledge of the market. Then one firm is labeled by $i=1$ and the other $i=2$. In game, players behave adaptively, following a bounded rationality adjustment process based on a local estimate of the marginal profit $\partial \Pi_{i} / \partial q_{i}$ (see $[2,3,6$, $8,9])$. For example, if a firm thinks the marginal profit of the time $t$ is positive, it is decided to increase its production of the time $t+1$ or to decrease its production if the marginal profit is negative. If the $i$ th firm at time $t$ is $q_{i}(t)$, its output at time $t+1$ can be modeled as

$$
q_{i}(t+1)=q_{i}(t)+\alpha_{i} q_{i}(t) \frac{\partial \Pi_{i}\left(q_{1}, q_{2}\right)}{\partial q_{i}}, \quad i=1,2,
$$

where $\Pi_{i}\left(q_{1}, q_{2}\right)$ is the after-tax profit of the $i$ th firmat time $t$ and $\alpha_{i}$ is positive parameter representing the speed of adjustment. As usual in duopoly models, the price $p$ of the good at time $t$ is determined by the total supply $Q(t)=$ $q_{1}(t)+q_{2}(t)$ through a demand function (see [3]):

$$
p=f(Q)=a-b Q,
$$

where $a$ and $b$ are positive constant, and $a$ is the highest price in the market. We assume that the production cost function has the nonlinear form:

$$
C_{i}\left(q_{i}\right)=c_{i}+d_{i} q_{i}+e_{i} q_{i}^{2}, \quad i=1,2,
$$

where the positive parameter $c_{i}$ is fixed cost of the $i$ th firm. In general, the cost function $C_{i}\left(q_{i}\right)$, climbing with the increase of the product output, is convex, so its first derivative $C_{i}^{\prime}\left(q_{i}\right)$ and second derivative $C_{i}^{\prime \prime}\left(q_{i}\right)$ are positive. We can assume that the parameters $d_{i}, e_{i}$ are positive. In order to make the duopoly game on the rails, the marginal profit of the $i$ th firm must be less than the highest price of the good in the market. Therefore, $d_{i}+2 e_{i} q_{i}<a, i=1,2$.

Hence the after-tax profit $\Pi_{i}$ of the $i$ th firm is given by

$$
\begin{array}{r}
\Pi_{i}=\left[q_{i}(t)(a-b Q(t))-\left(c+d_{i} q_{i}(t)+e_{i} q_{i}^{2}(t)\right)\right](1-r), \\
i=1,2,
\end{array}
$$

where $r$ is the tax rate of business income tax, and $0 \leq r<1$; $r=0$ represents pretax profit. The marginal profit of the $i$ th firm at the time $t$ is

$$
\begin{array}{r}
\frac{\partial \Pi_{i}}{\partial q_{i}}=\left(a-b Q(t)-b q_{i}(t)-d_{i}-2 e_{i} q_{i}(t)\right) \\
(1-r), \\
i=1,2 .
\end{array}
$$

The duopoly model with bounded rational players can be written in the form:

$$
\begin{aligned}
& q_{i}(t+1) \\
&= q_{i}(t)+\alpha_{i} q_{i}(t)\left[a-b Q(t)-\left(b+2 e_{i}\right) q_{i}(t)-d_{i}\right] \\
& \times(1-r), \quad i=1,2 .
\end{aligned}
$$

2.1. Equilibrium Points and Local Stability. In order to make the solution of the output duopoly model have the economical significance, we study the nonnegative stable state solution of the model in this paper. The equilibrium solution of the dynamics system (6) is the following algebraic nonnegative solution:

$$
\begin{aligned}
& q_{1}\left(a-b Q-\left(b+2 e_{1}\right) q_{1}\right)=0, \\
& q_{2}\left(a-b Q-\left(b+2 e_{2}\right) q_{2}\right)=0 .
\end{aligned}
$$

From (7), we can get four fixed points:

$$
\begin{gathered}
E_{0}=(0,0), \quad E_{1}=\left(\frac{a-d_{1}}{2 b+2 \mathrm{e}_{1}}, 0\right), \\
E_{2}=\left(0, \frac{a-d_{2}}{2 b+2 e_{2}}\right), \quad E^{*}=\left(q_{1}^{*}, q_{2}^{*}\right),
\end{gathered}
$$

where

$$
\begin{aligned}
& q_{1}^{*}=\frac{\left(a-d_{1}\right)\left(2 b+2 e_{2}\right)-b\left(a-d_{2}\right)}{3 b^{2}+4 b e_{1}+4 b e_{2}+4 e_{1} e_{2}}, \\
& q_{2}^{*}=\frac{\left(a-d_{2}\right)\left(2 b+2 e_{1}\right)-b\left(a-d_{1}\right)}{3 b^{2}+4 b e_{1}+4 b e_{2}+4 e_{1} e_{2}} .
\end{aligned}
$$

Since $E_{0}, E_{1}$, and $E_{2}$ are on the boundary of the decision set, $J=\left\{\left(q_{1}, q_{2}\right) \mid q_{1} \geq 0, q_{2} \geq 0\right\}$, they are called boundary equilibriums. $E^{*}$ is the unique Nash equilibrium provided that

$$
\begin{aligned}
& \left(a-d_{1}\right)\left(b+2 e_{2}\right)-b\left(d_{1}-d_{2}\right)>0, \\
& \left(a-d_{2}\right)\left(b+2 e_{1}\right)-b\left(d_{2}-d_{1}\right)>0 .
\end{aligned}
$$


The Nash equilibrium $E^{*}$ is located at the intersection of the two reaction curves which represent the locus of points of vanishing marginal profits in (5). In the following, we assume that (10) is satisfied, so the Nash equilibrium $E^{*}$ exists.

The study of the local stability of equilibrium points is based on the eigenvalues of the Jacobian matrix of the (6):

$$
\mathbf{J}=\left[\begin{array}{cc}
1+\alpha_{1} A_{1} & -\alpha_{1} b(1-r) q_{1} \\
-\alpha_{2} b(1-r) q_{2} & 1+\alpha_{2} A_{2}
\end{array}\right]
$$

where

$$
\begin{aligned}
& A_{1}=\left(a-\left(4 b+4 e_{1}\right) q_{1}-b q_{2}-d_{1}\right)(1-r), \\
& A_{2}=\left(a-b q_{1}-\left(4 b+4 e_{2}\right) q_{2}-d_{2}\right)(1-r) .
\end{aligned}
$$

As regards the conditions for the fixed point to be stable (see $[2,3]$ ), we have the following result.

Theorem 1. The boundary equilibria $E_{0}, E_{1}$, and $E_{2}$ are unstable equilibrium points.

Proof. At the boundary fixed point $E_{0}$, the Jacobian matrix is

$$
\mathbf{J}\left(E_{0}\right)=\left[\begin{array}{cc}
1+\alpha_{1}\left(a-d_{1}\right)(1-r) & 0 \\
0 & 1+\alpha_{2}\left(a-d_{2}\right)(1-r)
\end{array}\right] .
$$

The eigenvalues of $\mathbf{J}\left(E_{0}\right)$ are $\lambda_{1}=1+\alpha_{1}\left(a-d_{1}\right)(1-r)$ and $\lambda_{2}=1+\alpha_{2}\left(a-d_{2}\right)(1-r)$, which are greater than unity. Thus $E_{0}$ is a repelling node with eigendirections along the coordinate axes $q_{1}$ and $q_{2}$.

At the boundary fixed point $E_{1}$, the Jacobian matrix becomes

$$
\mathbf{J}\left(E_{1}\right)=\left[\begin{array}{cc}
1-\alpha_{1}\left(a-d_{1}\right)(1-r) & -\frac{\alpha_{1} b\left(a-d_{1}\right)(1-r)}{2 b+2 e_{1}} \\
0 & 1+\frac{\alpha_{2}\left(\left(a-d_{2}\right)\left(b+2 e_{1}\right)-b\left(d_{2}-d_{1}\right)\right)(1-r)}{2 b+2 e_{1}}
\end{array}\right]
$$

whose eigenvalues are given by $\lambda_{1}=1-\alpha_{1}\left(a-d_{1}\right)(1-r)$ with eigenvector $\xi_{1}=(1,0)$ along $q_{1}$ axe and $\lambda_{2}=1+\left(\alpha_{2}\left(\left(a-d_{2}\right)-\right.\right.$ $\left.b\left(d_{2}-d_{1}\right)\left(b+2 e_{1}\right)(1-r)\right) /\left(2 b+2 e_{1}\right)$ with eigenvector $\xi_{2}=([(1-$ $\left.\left.r)\left(2 b+2 e_{1}\right)+\alpha_{2}\left(\left(a-d_{2}\right)\left(b+2 e_{1}\right)-b\left(d_{2}-d_{1}\right)\right)\right] / \alpha_{1} b\left(a-d_{1}\right), 1\right)$, thus if $\alpha_{1}<2 /\left[\left(a-d_{1}\right)(1-r)\right], E_{1}$ is saddle point, with local stable manifold along $q_{1}$ axis and the unstable tangent to $\xi_{2}$. Otherwise, $E_{1}$ is an unstable node.

The bifurcation occurring at $\alpha_{1}=2 /\left[\left(a-d_{1}\right)(1-r)\right]$ is a flip bifurcation at which $E_{1}$ from attracting becomes repelling along $q_{1}$ axis, on which a cycle of period 2 appears.

From the similarity between $E_{1}$ and $E_{2}, E_{2}$ is a saddle point with local stable manifold along $q_{2}$ axis and the unstable one tangent to $\xi_{1}=\left(1,\left[(1-r)\left(2 b+2 e_{2}\right)+\alpha_{1}\left(\left(a-d_{1}\right)(b+\right.\right.\right.$ $\left.\left.\left.\left.2 e_{2}\right)-b\left(d_{1}-d_{2}\right)\right)\right] / \alpha_{2} b\left(a-d_{2}\right)\right)$, if $\alpha_{2}<2 /\left[\left(a-d_{2}\right)(1-r)\right], E_{2}$ is saddle point, with local stable manifold along $q_{2}$ axis and the unstable tangent to $\xi_{1}$. Otherwise, it is an unstable node. Hence Theorem 1 is true.

In order to study the local stability of Nash equilibrium $E^{*}=\left(q_{1}^{*}, q_{2}^{*}\right)$, we estimate the Jacobian matrix at $E^{*}$, which is

$$
\begin{aligned}
& \mathbf{J}\left(E^{*}\right) \\
& =\left[\begin{array}{cc}
1-2 \alpha_{1}\left(b+e_{1}\right) q_{1}^{*}(1-r) & -\alpha_{1} b q_{1}^{*}(1-r) \\
-\alpha_{2} b q_{2}^{*}(1-r) & 1-2 \alpha_{2}\left(b+e_{2}\right) q_{2}^{*}(1-r)
\end{array}\right] .
\end{aligned}
$$

The characteristic equation is

$$
P(\lambda)=\lambda^{2}-\operatorname{Tr} \lambda+\operatorname{Det}=0,
$$

where $\operatorname{Tr}$ is the trace and Det is the determinant, and

$$
\begin{gathered}
\operatorname{Tr}=2-2 f_{1} \alpha_{1}-2 f_{2} \alpha_{2}, \\
\text { Det }=1-2 f_{1} \alpha_{1}-2 f_{2} \alpha_{2}+f_{3} \alpha_{1} \alpha_{2},
\end{gathered}
$$

where $f_{1}=\left(b+e_{1}\right) q_{1}^{*}(1-r)$ is positive, $f_{2}=\left(b+e_{2}\right) q_{2}^{*}(1-r)$ is positive, and $f_{3}$ equals $4 f_{1} f_{2}+b^{2} q_{1}^{*} q_{2}^{*}(1-r)^{2}$, is positive.

Since

$$
\begin{aligned}
& \operatorname{Tr}^{2}-4 \text { Det } \\
& \quad=4\left[f_{2} \alpha_{2}-f_{1} \alpha_{1}\right]^{2}+4\left(4 f_{1} f_{2}-f_{3}\right) \alpha_{1} \alpha_{2}>0,
\end{aligned}
$$

the eigenvalues of Nash equilibrium are real. The local stability of Nash equilibrium is given by Jury's condition (see $[2,3,6,7])$, which are

(a) $1-\operatorname{Tr}+$ Det $=f_{3} \alpha_{1} \alpha_{2}>0$,

(b) $1+\operatorname{Tr}+$ Det $>0$.

The first condition is satisfied and the second condition becomes

$$
4 f_{1} \alpha_{1}+4 f_{2} \alpha_{2}-f_{3} \alpha_{1} \alpha_{2}-4<0 \text {. }
$$

This equation defines a region of stability in the plane of the speeds of adjustment $\left(\alpha_{1}, \alpha_{2}\right)$. The stability region is bounded by the portion of hyperbola with positive $\alpha_{1}$ and $\alpha_{2}$, whose equation is

$$
4 f_{1} \alpha_{1}+4 f_{2} \alpha_{2}-f_{3} \alpha_{1} \alpha_{2}-4=0 .
$$

For the values of $\left(\alpha_{1}, \alpha_{2}\right)$ inside the stability region, the Nash equilibrium $E^{*}$ is stable and loses its stability through 
a period doubling (flip) bifurcation. The bifurcation curve determined by (20) intersects the axes $\alpha_{1}$ and $\alpha_{2}$, respectively, whose coordinates are given by

$$
S_{1}=\left(\frac{1}{f_{1}}, 0\right), \quad S_{2}=\left(0, \frac{1}{f_{2}}\right) .
$$

Therefore, from the previously mentioned derivation, we have following theorem illustrating the local stability of equilibrium $E^{*}$.

Theorem 2. The stable region of equilibrium $E^{*}$ is enclosed by hyperbola defined by (20) and the axes $\alpha_{1}$ and $\alpha_{2}$.

Theorem 2 and (6) indicate that when the adjusting speed of $\alpha_{1}$ and $\alpha_{2}$ of the two firms' production is in the area defined by (20) and the axes $\alpha_{1}, \alpha_{2}$, the output of the two firms will tend towards the equilibrium point $E^{*}$. The maximum profit will be obtained at this point, just as

$$
\begin{aligned}
\Pi_{i}^{*}= & {\left[q_{i}^{*}\left(a-b Q^{*}\right)-\left(c_{i}+d_{i} q_{i}^{*}+e_{i} q_{i}^{2}\right)\right] } \\
& \times(1-r), \quad i=1,2,
\end{aligned}
$$

where $Q^{*}=q_{1}^{*}+q_{2}^{*}$.

It is noticeable that the game is based on the bounded rationality. The two firms cannot reach the Nash equilibrium at once. They may reach the equilibrium point after rounds of games. But once one player or both players adjust the production too fast and push $\alpha_{1}, \alpha_{2}$ beyond the bifurcation curve (defined by (20)), the system becomes unstable.

Similar argument applies if the parameters $\alpha_{1}$ and $\alpha_{2}$ are fixed parameters and the parameters $d_{1}, d_{2}$ are varied.

2.2. Numerical Simulation. We can show the stability of the Nash equilibrium point $E^{*}$ through the numerical experiments and show the way of the system to the chaos through period doubling bifurcation. The parameters have taken the values $\alpha_{1}=0.1, a=10, b=1, c_{1}=1.1, c_{2}=1$, $d_{1}=1, d_{2}=1, e_{1}=1, e_{2}=1.1$, and $r=0.3$. Figure $1(\mathrm{a})$ shows that the bifurcation diagram of the system (6) is convergent to Nash equilibrium for $\alpha_{2}<0.3901$. Then if $\alpha_{2}>$ 0.3901 , Nash equilibrium becomes unstable. Period doubling bifurcations appears, and finally chaotic behaviors occur. Also the maximum Lyapunov exponent (Lyap.) is plotted. Positive values of Lyapunov exponent show that the solution has chaotic behavior. Taking the corresponding output on the output trace in (4), the profit trace bifurcation diagram can be drawn (as shown by Figure 1(b)). In Figure 1(b), $\Pi_{1}$ represents the profit of the $i=$ first firm, and $\Pi_{2}$ represents the profit of the $i=$ second firm. We can see the change of profit is the same as the output of the player with the variance of parameter $\alpha_{1}$. Figures 1(a) and 1(b) also show that if the firm does the decision making more carefully, the output and the profit will be more stable. Figure 1(c) shows strange attractor for the values of the parameters $a=10, b=1, d_{1}=1, d_{2}=1, e_{1}=1$, $e_{2}=1.1, r=0.3, \alpha_{1}=0.1$, and $\alpha_{2}=0.54$. Strange attractors of the system (6) exhibit a fractal structure. Figure 1(d) shows the region of stability of the Nash equilibrium for the values of the parameters $a=10, b=1, d_{1}=1, d_{2}=1, e_{1}=1, e_{2}=1.1$, and $r=0.3$. Equation (20) and the economical significance of parameters (which is positive here) define the regions of stability in the plane of adjustment $\left(\alpha_{1}, \alpha_{2}\right)$.

\section{The Output Duopoly Game Having Output Lower Limiters}

Next, we assume that the $i$ th firm will impose lower limiter $q_{i}^{\min }$ on output for economies of scale, and (6) becomes

$$
\begin{array}{r}
q_{i}(t+1)=\operatorname{Max}\left[f_{i}\left(q_{1}(t), q_{2}(t)\right), q_{i}^{\min }\right], \\
i=1,2,
\end{array}
$$

where $q_{i}^{\min }>0$ and

$$
\begin{array}{r}
f_{i}\left(q_{1}, q_{2}\right)=q_{i}+\alpha_{i} q_{i}\left[a-b Q-\left(b+2 e_{i}\right) q_{i}-d_{i}\right](1-r), \\
i=1,2 .
\end{array}
$$

Limiting the output is economically justified in the real world. It can be explained by capacity constraints, financial constraints, breakeven, and steadiness.

3.1. Equilibrium Points. In order to study the qualitative behavior of the solutions of the nonlinear map (23), we define the equilibrium points of the dynamic duopoly game by letting

$$
q_{i}(t+1)=q_{i}(t), \quad i=1,2,
$$

where $q_{i}(t+1)$ is determined by (23). Comparing $f_{i}\left(q_{1}(t), q_{2}(t)\right.$ ) (in brief, $f_{i}(t)$, defined by $(24)$ ) with $q_{i}^{\text {min }}$, $i=1,2$, there are four cases.

(a) $f_{1}(t) \geq q_{1}^{\min }$ and $f_{2}(t) \geq q_{2}^{\min }$. When $q_{1}^{\min } \leq q_{1}^{*}$ and $q_{2}^{\min } \leq q_{2}^{*}$, the solution of the system (23) gives one fixed point:

$$
E^{*}=\left(q_{1}^{*}, q_{2}^{*}\right)
$$

where $q_{1}^{*}=\left(\left(a-d_{1}\right)\left(2 b+2 e_{2}\right)-b\left(a-d_{2}\right)\right) /\left(3 b^{2}+\right.$ $\left.4 b e_{1}+4 b e_{2}+4 e_{1} e_{2}\right), q_{2}^{*}=\left(\left(a-d_{2}\right)\left(2 b+2 e_{1}\right)-b(a-\right.$ $\left.\left.d_{1}\right)\right) /\left(3 b^{2}+4 b e_{1}+4 b e_{2}+4 e_{1} e_{2}\right)$, and $E^{*}$ is the unique Nash equilibrium when (10) is satisfied.

(b) $f_{1}(t)<q_{1}^{\min }$ and $f_{2}(t)>q_{2}^{\min }$. When $q_{1}^{\min }>q_{1}^{*}$ and $q_{2}^{\min } \leq q_{20}^{*}$, we can obtain a fixed point:

$$
F_{1}=\left(q_{1}^{\min }, q_{20}^{*}\right)
$$

where $q_{20}^{*}=\left(a-b q_{1}^{\min }-d_{2}\right) /\left(2 b+2 e_{2}\right)$.

(c) $f_{1}(t)>q_{1}^{\min }$ and $f_{2}(t)<q_{2}^{\min }$. When $q_{1}^{\min } \leq q_{10}^{*}$ and $q_{2}^{\min }>q_{2}^{*}$, we have one fixed point:

$$
F_{2}=\left(q_{10}^{*}, q_{2}^{\min }\right)
$$

where $q_{10}^{*}=\left(a-b q_{2}^{\min }-d_{1}\right) /\left(2 b+2 e_{1}\right)$. 


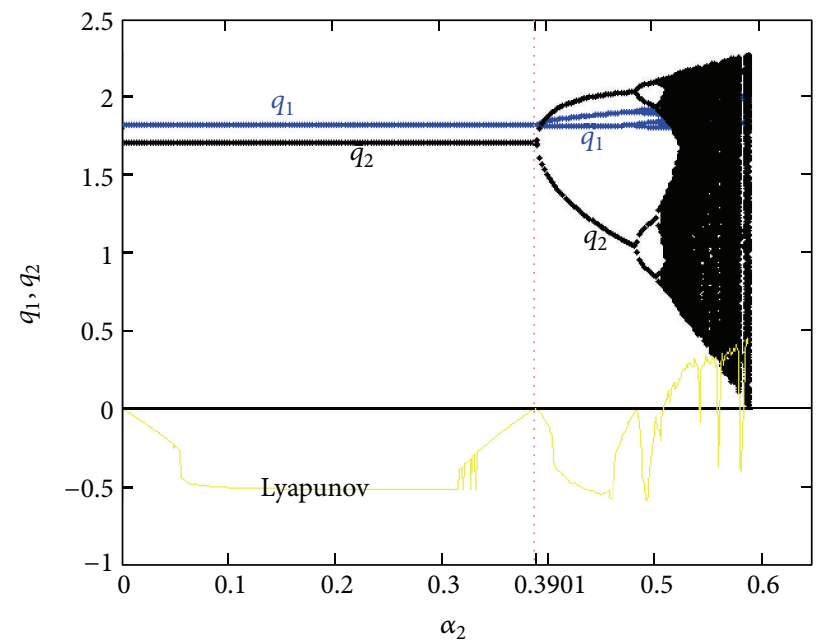

(a)

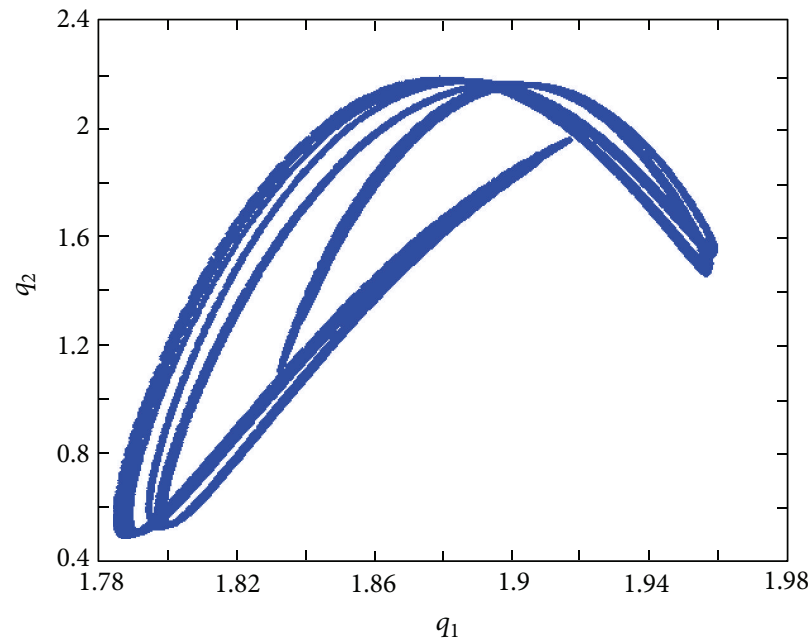

(c)

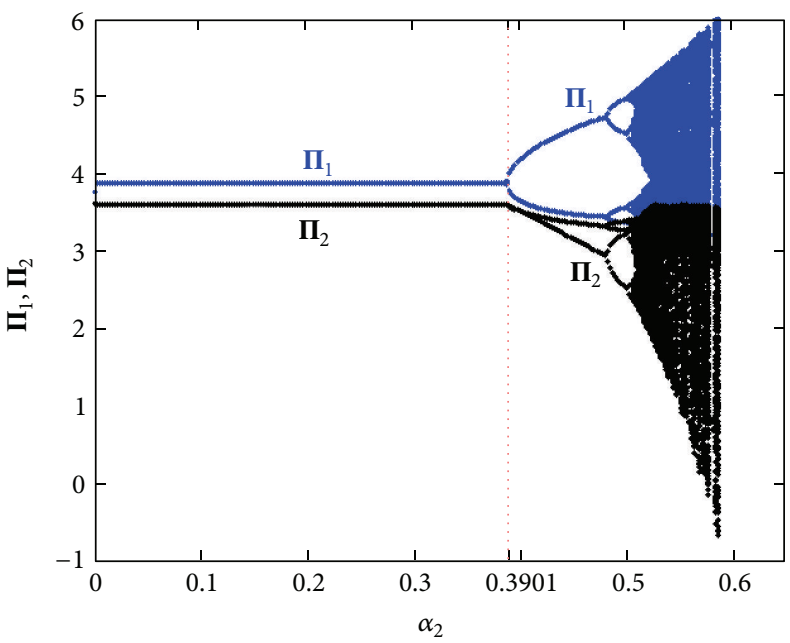

(b)

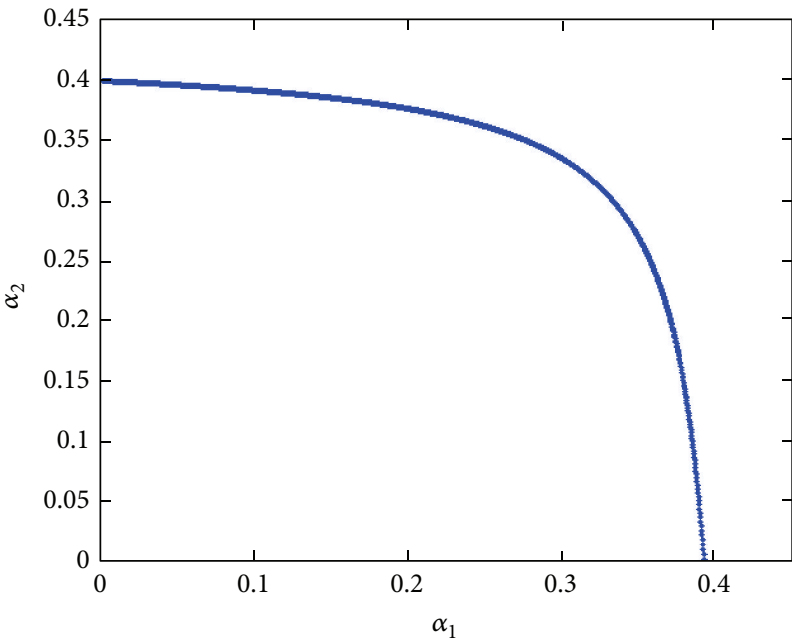

(d)

FIgURE 1: Partial numerical simulation of the system (6). (a) The bifurcation diagram of the evolution of output. (b) The bifurcation diagram of the evolution of after-tax profit. (c) The strange attractor. (d) The stable region of Nash equilibrium of duopoly game in the phase plane of speed of adjustment.

(d) $f_{1}(t)<q_{1}^{\min }$ and $f_{2}(t)<q_{2}^{\min }$. When $a-b\left(q_{1}^{\min }+\right.$ $\left.q_{2}^{\text {min }}\right)-\left(b+2 e_{i}\right) q_{i}^{\text {min }}-d_{i} \leq 0, i=1,2$, the solution of the system (23) gives one fixed point:

$$
F_{3}=\left(q_{1}^{\min }, q_{2}^{\min }\right) \text {. }
$$

From the previously mentioned analysis, we can see that the existence of equilibriums $E^{*}, F_{1}, F_{2}$, or $F_{3}$ has relation to the size of lower limiters $q_{1}^{\min }$ and $q_{2}^{\min }$, so we call them conditional equilibrium points. The relations between existence of equilibrium and lower limiters are summarized in Figure 2. In Figure 2, EF $\left(a-b q_{1}^{\min }-b q_{2}^{\min }=0\right)$ denotes the maximal capacity of market, where the price of the good comes up to zero. When $\left(q_{1}^{\min }, q_{2}^{\min }\right)$ is located in region $\mathbf{I}$, which is enclosed by horizontal line $\mathrm{BN}\left(q_{2}^{\min }=q_{2}^{*}\right)$, vertical line $\operatorname{AN}\left(q_{1}^{\min }=q_{1}^{*}\right)$, and the axes $q_{i}^{\text {min }}, i=1,2$, the system (23) has Nash equilibrium $E^{*}$. If $\left(q_{1}^{\min }, q_{2}^{\min }\right)$ falls in region
II, which is surrounded by line AN, line NC $\left(a-b\left(q_{1}^{\min }+\right.\right.$ $\left.\left.q_{2}^{\min }\right)-\left(b+2 e_{2}\right) q_{2}^{\min }-d_{2}=0\right)$, and the axis $q_{1}^{\min }$, the system (23) gives the conditional equilibrium $F_{1}$. If it is located in region III, which is enclosed by line $\mathrm{NC}$, line $\mathrm{EF}$, line $\mathrm{DN}$ $\left(a-b\left(q_{1}^{\min }+q_{2}^{\min }\right)-\left(b+2 e_{1}\right) q_{1}^{\min }-d_{1}=0\right)$, and the axes $q_{i}^{\min }, i=1,2$, equilibrium point of the system (23) is $F_{3}$. If it is situated in region IV surrounded with line BN, line DN, and the axis $q_{2}^{\mathrm{min}}$, conditional equilibrium point of the system (23) becomes $F_{2}$.

It is very interesting that conditional equilibrium $F_{1}$ perches on line $\mathrm{NC}, F_{2}$ stands on line $\mathrm{DN}$, and $F_{3}$ is situated in region III. Furthermore, the feasible region of conditional equilibrium points consists of region III, and its boundary is convex. What is more, Nash equilibrium $E^{*}$ (namely, $N$ in Figure 2) is one of the vertices of the region. So the equilibria of the system (23) are different from those of the system (6) which only has boundary equilibria and Nash equilibrium. 


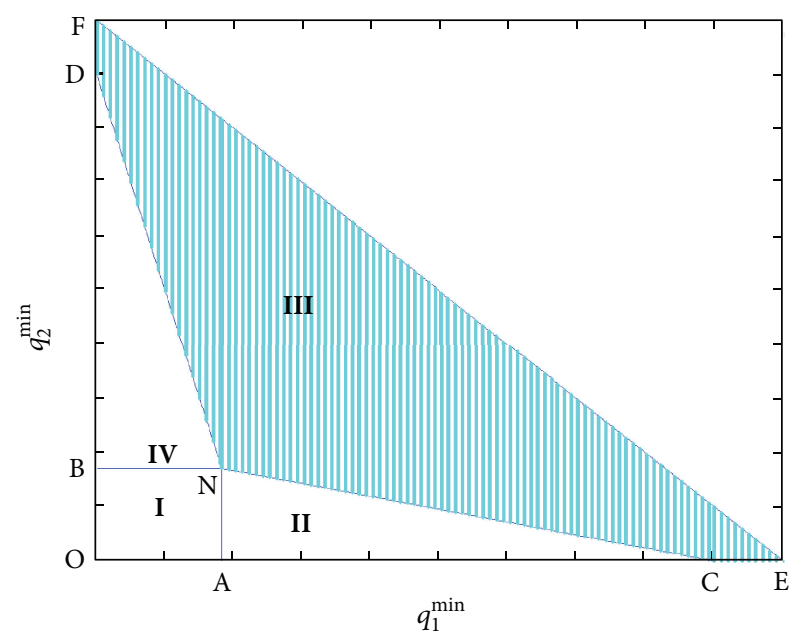

FIGURE 2: The distributions of conditional equilibrium points of the system (23), at parameter values $\left(a, b, d_{1}, d_{2}, e_{1}, e_{2}\right)=$ $(10,1,1,1,1,1.1)$.

3.2. Stability. Using the method similar to Section 2.1, we can draw the conclusion that Nash equilibrium $E^{*}$ of the system (23) has the same stable region in the plane of the speeds of adjustment $\left(\alpha_{1}, \alpha_{2}\right)$ as system (6). That is to say, its region of stability is bounded by (20) and axes $\alpha_{i}, i=1,2$. Although the equilibrium of the system (23) may still be looked as a result of "learning" and "evolution," the adjustment of production is restrained due to lower limiter. Moreover, size of limiters influences existence of Nash equilibrium.

In the following, we will explore the stability of conditional equilibrium points $F_{1}, F_{2}$, or $F_{3}$ of the system (23), respectively.

As for conditional equilibrium $F_{3}$ of the system (23), the following result can be made.

Theorem 3. The conditional equilibrium $F_{3}$ of the system (23) is globally stable in the plane of the speeds of adjustment $\left(\alpha_{1}, \alpha_{2}\right)$.

Proof. Because $q_{i}^{\mathrm{min}}, i=1,2$ are positive and the lower limiters of output,

$$
q_{i}(t) \geq q_{i}^{\min }, \quad i=1,2, t=0,1,2, \ldots
$$

As Section 3.1 shows, when conditional equilibrium $F_{3}$ exists, $q_{i}^{\mathrm{min}}, i=1,2$ must satisfy

$$
\begin{array}{r}
a-b\left(q_{1}^{\min }+q_{2}^{\min }\right)-\left(b+2 e_{i}\right) q_{i}^{\min }-d_{i} \leq 0, \\
i=1,2 .
\end{array}
$$

Note that $b, d_{1}, d_{2}, e_{1}$, and $e_{2}$ are positive; also note that when $q_{i}(t) \geq q_{i}^{\min }$, we have
(a) $a-b\left(q_{1}(t)+q_{2}(t)\right)-\left(b+2 e_{1}\right) q_{1}(t)-d_{1} \leq 0$,
(b) $a-b\left(q_{1}(t)+q_{2}(t)\right)-\left(b+2 e_{2}\right) q_{2}(t)-d_{2} \leq 0$.

Now, when one of the previously mentioned two inequalities (a) and (b) becomes an equation, we prove that

$$
q_{i}(t)=q_{i}^{\min }, \quad i=1,2 .
$$

Using the method of reduction to absurdity can prove this conclusion. We now assume that there is one strict inequality at least for $q_{1}(t) \geq q_{1}^{\min }$ and $q_{2}(t) \geq q_{2}^{\min }$, for example $q_{1}(t)>$ $q_{1}^{\min }$. If $a-b\left(q_{1}(t)+q_{2}(t)\right)-\left(b+2 e_{i}\right) q_{i}(t)-d_{i}=0, i=1$ or 2 , then

$$
\begin{array}{r}
a-b\left(q_{1}^{\min }+q_{2}^{\min }\right)-\left(b+2 e_{i}\right) q_{i}^{\min }-d_{i}>0, \\
i=1 \text { or } 2,
\end{array}
$$

which is impossible. Hence if there is an equation in

$$
\begin{array}{r}
a-b\left(q_{1}(t)+q_{2}(t)\right)-\left(b+2 e_{i}\right) q_{i}(t)-d_{i} \leq 0, \\
i=1,2 .
\end{array}
$$

then $q_{i}(t)=q_{i}^{\min }, i=1,2$.

If $a-b\left(q_{1}(t)+q_{2}(t)\right)-\left(b+2 e_{i}\right) q_{i}(t)-d_{i}<0, i=1$ and 2 , according to condition (a), condition (b), and the map (23), we can obtain two series $q_{1}(t)$ and $q_{2}(t)(t=0,1,2, \ldots$,$) which$ descend monotonously and have lower bound $q_{1}^{\min }$ and $q_{2}^{\min }$, respectively. Therefore, they have limits when $t \rightarrow+\infty$. We assume that the limits are $q_{i 0}^{l}, i=1,2$, then $q_{i 0}^{l} \geq q_{i}^{\min }, i=1,2$. When there is an inequality at least in $q_{i 0}^{l} \geq q_{i}^{\min }, i=1,2$, we assume that $q_{10}^{l}>q_{1}^{\mathrm{min}}$ and $q_{11}^{l}$ is the image of $q_{10}^{l}$ following the map (23). Note that

$$
\begin{array}{r}
a-b\left(q_{10}^{l}(t)+q_{20}^{l}(t)\right)-\left(b+2 e_{i}\right) q_{i 0}^{l}(t)-d_{i}<0, \\
i=1,2 .
\end{array}
$$

Then $q_{11}^{l}<q_{10}^{l}$, which is a conflict with the fact that $q_{10}^{l}$ is the limit of series $q_{1}(t)$.

Hence, $q_{i}(t) \rightarrow q_{i}^{\min }, i=1,2$. We can know that Theorem 3 is true.

The global stability of $F_{3}$ indicates that when lower limiters increase to a certain extent, chaotic state of the output game disappears. In real business world, firms may suppress chaos and reduce volatility of output and profit by imposing lower limiter.

Considering the symmetry of the system (23), and conditional equilibrium points $F_{1}$ and $F_{2}$, we only need to discuss the stability of $F_{1}$. It is very difficult to infer the stable region of $F_{1}$. Here, we use numerical experiment to show the impact of lower limiters on regions of stability of $F_{1}$, which is illustrated in Figure 3. The parameter setting is in Figure 1(d). Figure 3 displays that magnitudes of lower limiters and size of initial output all have great influence on stability of $F_{1}$. As initial output lessens, the stable region of $F_{1}$ reduces.

In Figures 3(a) and 3(c), scope of $\alpha_{1}$ is drawn partly. In fact, we have the following theorem illustrating this.

Theorem 4. In the plane of the speeds of adjustment $\left(\alpha_{1}, \alpha_{2}\right)$, when initial output of the $i=$ second firm satisfies $q_{2}(0) \geq(a-$ $\left.\left(2 b+2 e_{1}\right) q_{1}^{\mathrm{min}}-d_{1}\right) / b$, the scope of $\alpha_{1}$ in the stable region of conditional equilibrium $F_{1}$ of the system (23) is $\alpha_{1}>0$. 


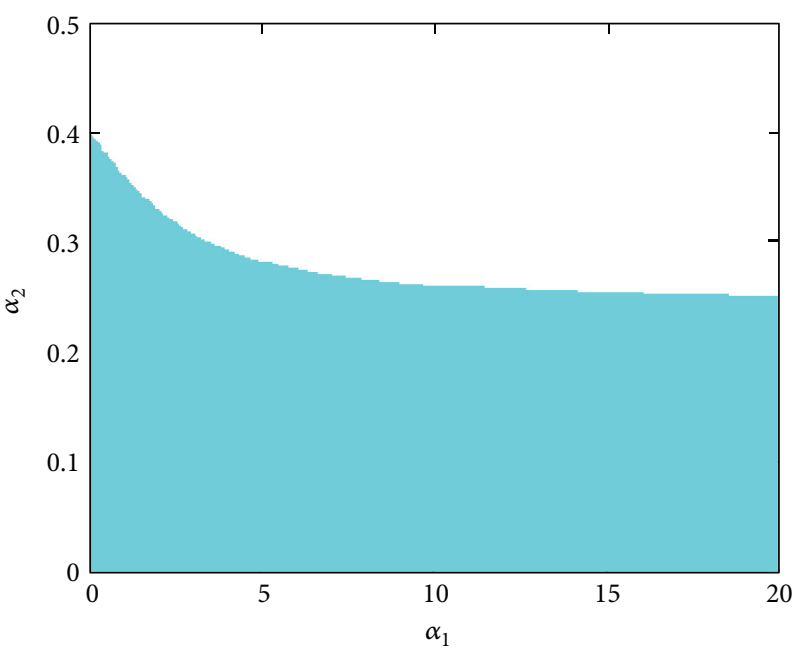

(a) $q_{1}^{\min }=1.832, q_{2}^{\min }=1.2, q_{1}(0)=1.86, q_{2}(0)=1.8$

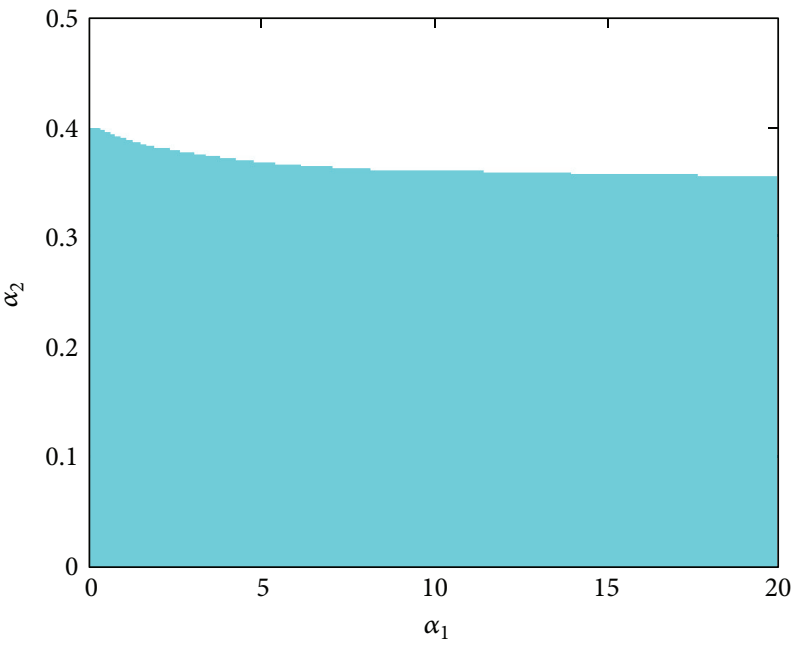

(c) $q_{1}^{\min }=1.846, q_{2}^{\min }=1.36, q_{1}(0)=1.86, q_{2}(0)=1.8$

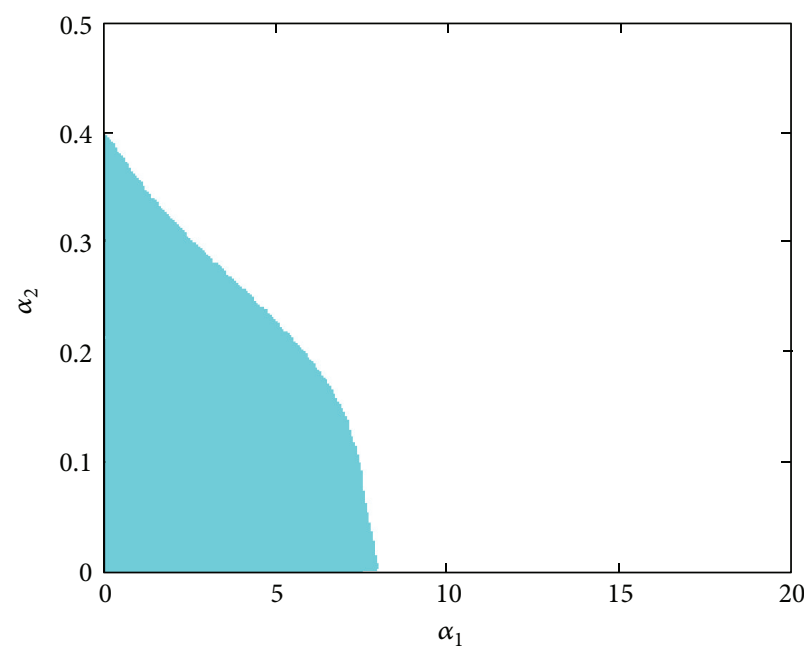

(b) $q_{1}^{\min }=1.832, q_{2}^{\min }=1.2, q_{1}(0)=1.86, q_{2}(0)=1.52$

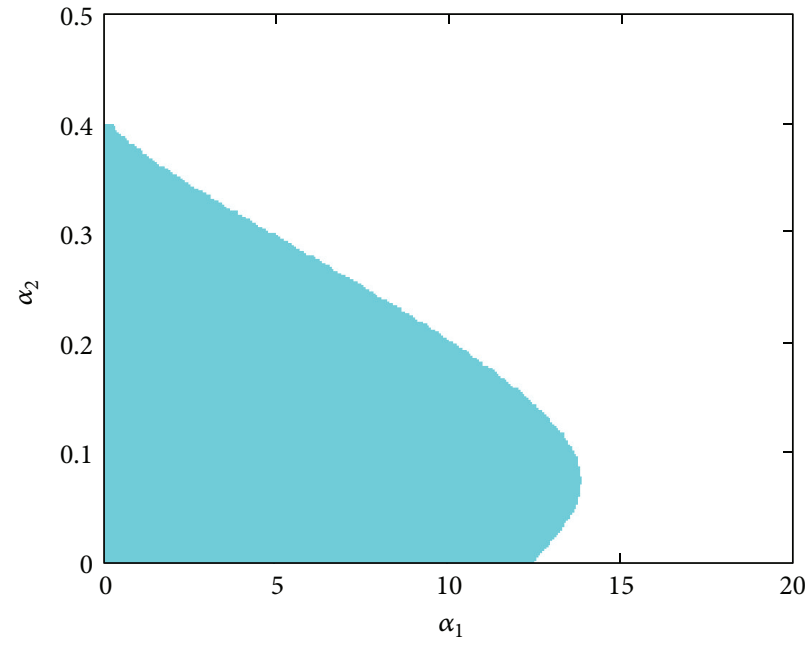

(d) $q_{1}^{\min }=1.846, q_{2}^{\min }=1.36, q_{1}(0)=1.86, q_{2}(0)=1.52$

FIgURE 3: The stable region of conditional equilibrium $F_{1}$ of the system (23).

Proof. Because $q_{1}^{\min }>q_{1}^{*}=\left(\left(a-d_{1}\right)\left(2 b+2 e_{2}\right)-b(a-\right.$ $\left.\left.d_{2}\right)\right) /\left(3 b^{2}+4 b e_{1}+4 b e_{2}+4 e_{1} e_{2}\right)$, so

$$
\frac{a-b q_{1}^{\min }-d_{2}}{2 b+2 e_{2}}>\frac{a-\left(2 b+2 e_{1}\right) q_{1}^{\min }-d_{1}}{b} .
$$

Note that the trajectories of output converge to $\left(q_{1}^{\min },(a-\right.$ $\left.\left.b q_{1}^{\min }-d_{2}\right) /\left(2 b+2 e_{2}\right)\right)$. If $q_{2}(0) \geq\left(a-\left(2 b+2 e_{1}\right) q_{1}^{\min }-d_{1}\right) / b$, as long as the speed of adjustment of the $i=$ second firm, $\alpha_{2}$ is small enough, after finite iterative time, we can obtain

$$
q_{2}(t)>\frac{a-\left(2 b+2 e_{1}\right) q_{1}^{\min }-d_{1}}{b} .
$$

That is to say, $a-\left(2 b+2 e_{1}\right) q_{1}^{\min }-b q_{2}(t)-d_{1}<0$.

Note that $q_{1}(t) \geq q_{1}^{\min }$. Therefore, given $\alpha_{1}>0$ at will, we have

$$
a-\left(2 b+2 e_{1}\right) q_{1}(t)-b q_{2}(t)-d_{1}<0 .
$$

According to the system (23), we can obtain a series of $q_{1}(t)(t=0,1,2, \ldots)$ which descend monotonously and have lower bound $q_{1}^{\min }$ except initial finite terms. Thus, they have limit when $t \rightarrow+\infty$. We assume that the limit is $q_{10}^{l}$; then $q_{10}^{l} \geq q_{1}^{\min }$. If $q_{10}^{l}>q_{1}^{\min }$, we assume that $q_{11}^{l}$ is the image of $q_{10}^{l}$ following the map (23). Note that

$$
a-b\left(q_{10}^{l}(t)+q_{2}(t)\right)-\left(b+2 e_{1}\right) q_{10}^{l}(t)-d_{1}<0 .
$$

Then $q_{11}^{l}<q_{10}^{l}$, which is a conflict with the fact that $q_{10}^{l}$ is the limit of series $q_{1}(t)$. Hence, $q_{10}^{l}=q_{1}^{\min }$. That is to say, if $q_{2}(0) \geq\left(a-\left(2 b+2 e_{1}\right) q_{1}^{\min }-d_{1}\right) / b$, the speed of adjustment of the $i=$ first firm has no effect on the stability of conditional equilibrium $F_{1}$. The proof is complete.

By the same way, we can obtain the following.

Theorem 5. In the plane of the speeds of adjustment $\left(\alpha_{1}, \alpha_{2}\right)$, when initial output of the $i=$ first firm satisfies $q_{1}(0) \geq(a-$ 


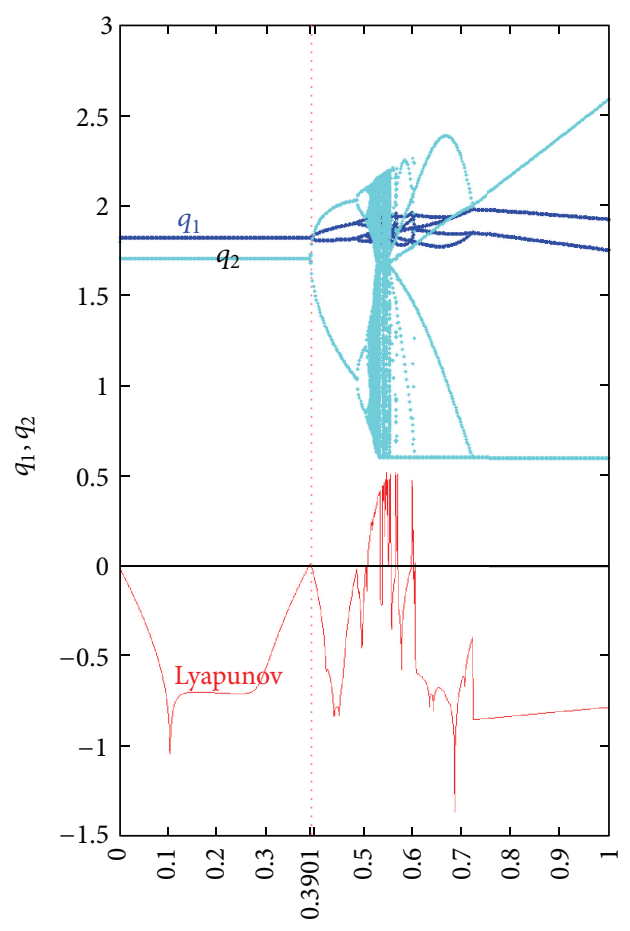

$\alpha_{2}$

(a)

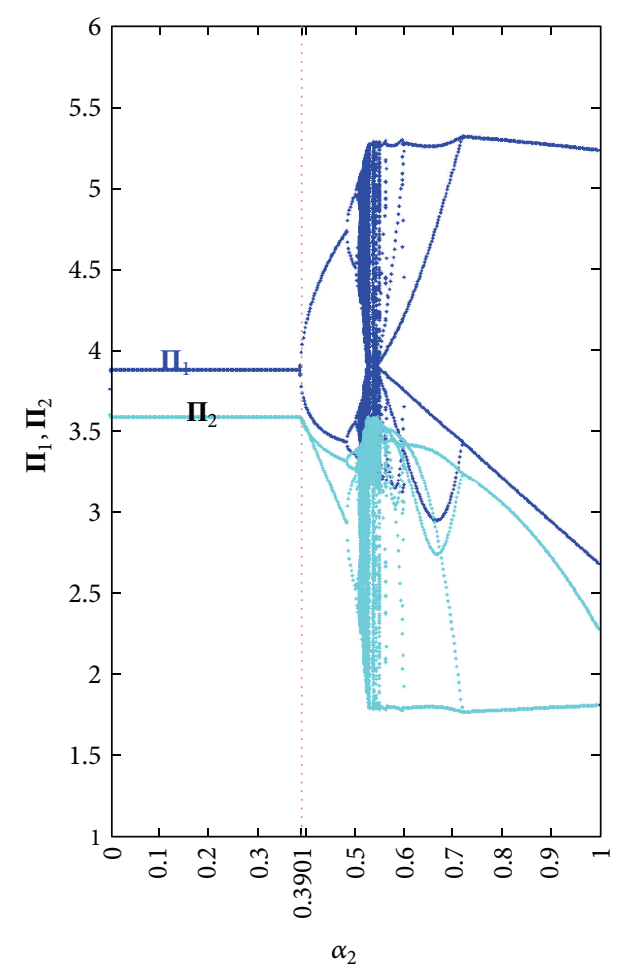

(b)

FIGURE 4: Bifurcation diagrams of the system (23) with output lower limiters $\left(q_{1}^{\min }, q_{2}^{\min }\right)=(0.4,0.6)$, at parameter values $\left(a, b, c_{1}, c_{2}, d_{1}, d_{2}, e_{1}, e_{2}, r, \alpha_{1},\right)=(10,1,1.1,1,1,1,1,1.1,0.3,0.1)$.

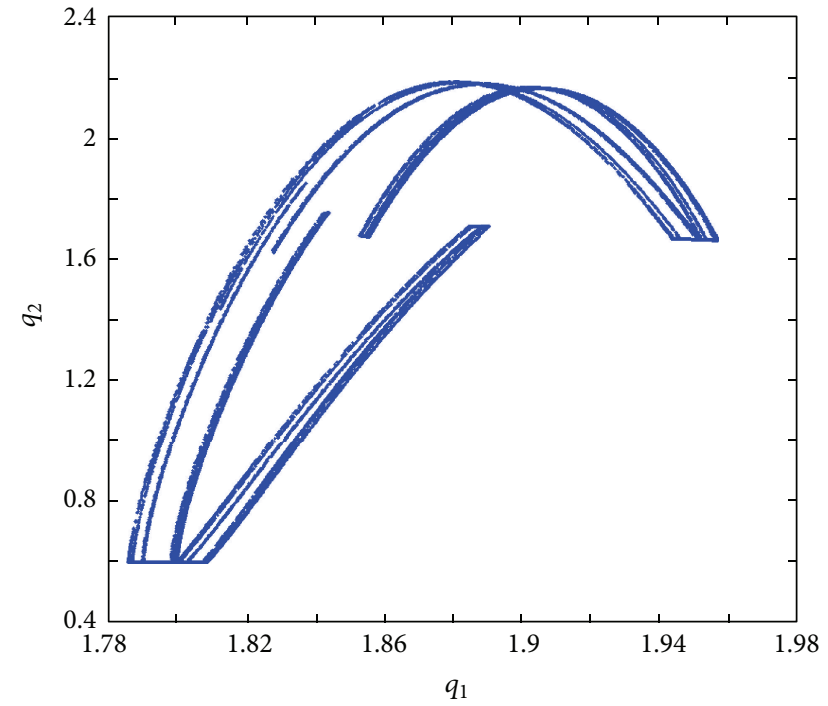

FIGURE 5: The strange attractor of the system (23) with output lower limiters $\left(q_{1}^{\mathrm{min}}, q_{2}^{\mathrm{min}}\right)=(0.4,0.6)$, at parameter values $\left(a, b, d_{1}, d_{2}, e_{1}, e_{2}, r, \alpha_{1}, \alpha_{2}\right)=(10,1,1,1,1,1.1,0.3,0.1,0.54)$.

$\left.\left(2 b+2 e_{2}\right) q_{2}^{\min }-d_{2}\right) / b$, the scope of $\alpha_{2}$ in the stable region of conditional equilibrium $F_{2}$ of the system (23) is $\alpha_{2}>0$.

3.3. Numerical Simulation. Numerical experiments are simulated to show the influence of lower limiters on the stability of Nash equilibrium, which are based on the same parameter setting as Figures 1(a) and 1(b).

Figure 4(a) reveals that the trajectories of output converges to the Nash equilibrium when $\alpha_{2}<0.3901$; for $\alpha_{2}>0.3901$, the Nash equilibrium becomes unstable, period doubling bifurcations appear, and chaotic behavior occurs. However, the dynamics of the map with lower limiters $\left(q_{1}^{\min }=0.4, q_{2}^{\min }=0.6\right)$ is different from that of the map without limiter. When $\alpha_{2}>0.6$, the period behaviors come forth again. Also the maximum Lyapunov exponent (lyap.) is plotted, where positive values indicate that the system has chaotic behaviors. Figure 4(b) based on the same parameter setting shows the impact of lower limiters on the evolution of profit. Comparing Figures 4(a) and 4(b) with Figures 1(a) and 1(b), respectively, we can see that imposing lower limiters can reduce fluctuations of production and profit.

Figure 5 shows the influence of production limiters on the strange attractor of the duopoly game. Comparing it with Figure 4(c), we can find the difference between the attractor of the system (6) and that of the system (23).

\section{Conclusions}

This paper is concerned with complex dynamics of duopoly game without and with output lower limiters. We discussed that if the behavior of producer is characterized by relatively low speeds of adjustment, the local production adjustment process without limiters converges to the unique Nash 
equilibrium. Complex behaviors such as cycles and chaos occur for higher values of speeds of adjustment.

Furthermore, we investigate how output lower limiters, which function identically to a recently explored chaos control method: phase space compression, and the limiter method, affect the output dynamics. The existence of Nash equilibrium becomes conditional. The distribution of the conditional equilibrium points is displayed in this paper, and their relation with lower limiter is studied. It is funny that the feasible region of conditional equilibrium points is convex and Nash equilibrium is one of the vertices of the region. We find that simple output lower limiters may (a) reduce the fluctuation of production and profit; (b) make chaos of the original duopoly game disappear; (c) help the firms to avoid the explosion of the economic system. The size of lower limiters and initial output also has relation with the stable region of conditional equilibrium points $F_{1}$ and $F_{2}$, and the relation is explored analytically and numerically. The conditional equilibrium points $\left(q_{1}^{\min }, q_{2}^{\min }\right)$ are globally stable in the plane of speeds of adjustment. The stability of the conditional points gives a theoretical basis for the phase space compression and the limiter method to control chaos in a special case.

\section{Acknowledgments}

This work was supported in part by the National Natural Science Foundation of China under Grants 71171099, 71073070, 71001028, and 70773051, by the National Social Science Foundation of China for major invitation-for-bid project under Grant 11\&ZD169, and by China Postdoctoral Science Foundation under Grant 20090461080. This work was also sponsored by Qing Lan Project and 333 Project of Jiangsu Province, Jiangsu University Top Talents Training Project, and Qatar National Priority Program NPRP 4-1162-1181 funded by Qatar National Research Fund.

\section{References}

[1] A. Cournot, Researches into the Mathematical Principles of the Theory of Wealth, Hachette, Paris, France, 1838.

[2] H. N. Agiza, A. S. Hegazi, and A. A. Elsadany, "Complex dynamics and synchronization of a duopoly game with bounded rationality," Mathematics and Computers in Simulation, vol. 58, no. 2, pp. 133-146, 2002.

[3] H. N. Agiza, A. S. Hegazi, and A. A. Elsadany, "The dynamics of Bowley's model with bounded rationality," Chaos, Solitons and Fractals, vol. 12, no. 9, pp. 1705-1717, 2001.

[4] H. N. Agiza and A. A. Elsadany, "Chaotic dynamics in nonlinear duopoly game with heterogeneous players," Applied Mathematics and Computation, vol. 149, no. 3, pp. 843-860, 2004.

[5] H. N. Agiza and A. A. Elsadany, "Nonlinear dynamics in the Cournot duopoly game with heterogeneous players," Physica A, vol. 320, no. 1-4, pp. 512-524, 2003.

[6] E. Ahmed and H. N. Agiza, "Dynamics of a Cournot game with n-competitors," Chaos, Solitons and Fractals, vol. 9, no. 9, pp. 1513-1517, 1998.
[7] E. Ahmed, H. N. Agiza, and S. Z. Hassan, "On modifications of Puu's dynamical duopoly," Chaos, Solitons and Fractals, vol. 11, no. 7, pp. 1025-1028, 2000.

[8] Z. H. Sheng, T. W. Huang, J. G. Du, Q. Mei, and H. Huang, "Study on self-adaptive proportional control method for a class of output models," Discrete and Continuous Dynamical Systems $B$, vol. 11, no. 2, pp. 459-477, 2009.

[9] M. T. Yassen and H. N. Agiza, "Analysis of a duopoly game with delayed bounded rationality," Applied Mathematics and Computation, vol. 138, no. 2-3, pp. 387-402, 2003.

[10] D. Rand, "Exotic phenomena in games and duopoly models", Journal of Mathematical Economics, vol. 5, no. 2, pp. 173-184, 1978.

[11] T. Puu, "Chaos in business cycles," Chaos, Solitons and Fractals, vol. 1, no. 5, pp. 457-473, 1991.

[12] T. Puu, "Complex dynamics with three oligopolists," Chaos, Solitons and Fractals, vol. 7, no. 12, pp. 2075-2081, 1996.

[13] M. Kopel, "Simple and complex adjustment dynamics in Cournot duopoly models," Chaos, Solitons and Fractals, vol. 7, no. 12, pp. 2031-2048, 1996.

[14] W. A. Brock and C. H. Hommes, "Heterogeneous beliefs and routes to chaos in a simple asset pricing model," Journal of Economic Dynamics and Control, vol. 22, no. 8-9, pp. 1235-1274, 1998.

[15] W. J. Den Haan, "The importance of the number of different agents in a heterogeneous asset-pricing model," Journal of Economic Dynamics and Control, vol. 25, no. 5, pp. 721-746, 2001.

[16] Z. W. Ding, Q. L. Hang, and L. X. Tian, "Analysis of the dynamics of Cournot team-game with heterogeneous players," Applied Mathematics and Computation, vol. 215, no. 3, pp. 10981105, 2009.

[17] T. Dubiel-Teleszynski, "Nonlinear dynamics in a heterogeneous duopoly game with adjusting players and diseconomies of scale," Communications in Nonlinear Science and Numerical Simulation, vol. 16, no. 1, pp. 296-308, 2011.

[18] Y. Q. Fan, T. Xie, and J. G. Du, "Complex dynamics of duopoly game with heterogeneous players: a further analysis of the output model," Applied Mathematics and Computation, vol. 218, no. 15, pp. 7829-7838, 2012.

[19] T. Onozaki, G. Sieg, and M. Yokoo, "Stability, chaos and multiple attractors: a single agent makes a difference," Journal of Economic Dynamics and Control, vol. 27, no. 10, pp. 1917-1938, 2003.

[20] J. X. Zhang, Q. L. Da, and Y. H. Wang, "Analysis of nonlinear duopoly game with heterogeneous players," Economic Modelling, vol. 24, no. 1, pp. 138-148, 2007.

[21] W. H. Huang, "Caution implies profit," Journal of Economic Behavior \& Organization, vol. 27, pp. 257-277, 1995.

[22] X.-Z. He and F. H. Westerhoff, "Commodity markets, price limiters and speculative price dynamics," Journal of Economic Dynamics and Control, vol. 29, no. 9, pp. 1577-1596, 2005.

[23] J. G. Du, T. G. Huang, Z. H. Sheng, and H. B. Zhang, "A new method to control chaos in an economic system," Applied Mathematics and Computation, vol. 217, no. 6, pp. 2370-2380, 2010.

[24] R. Stoop and C. Wagner, "Scaling properties of simple limiter control," Physical Review Letters, vol. 90, no. 15, Article ID 154101, 4 pages, 2003. 
[25] C. Wagner and R. Stoop, "Optimized chaos control with simple limiters," Physical Review E, vol. 63, Article ID 017201, 2 pages, 2000.

[26] X. Zhang and K. Shen, "Controlling spatiotemporal chaos via phase space compression," Physical Review E, vol. 63, pp. 4621246217, 2001. 


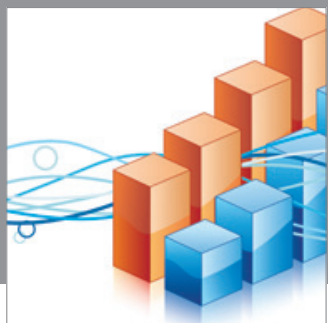

Advances in

Operations Research

mansans

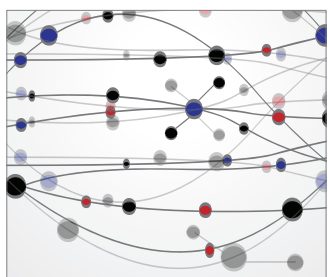

The Scientific World Journal
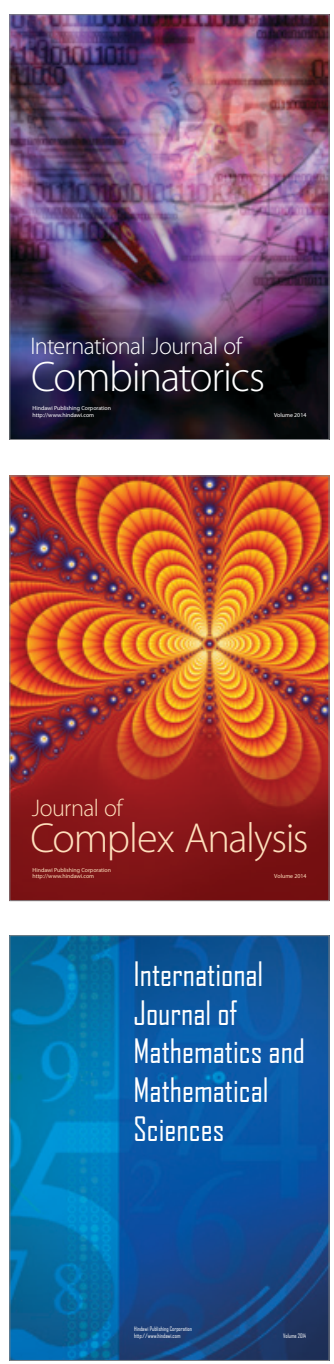
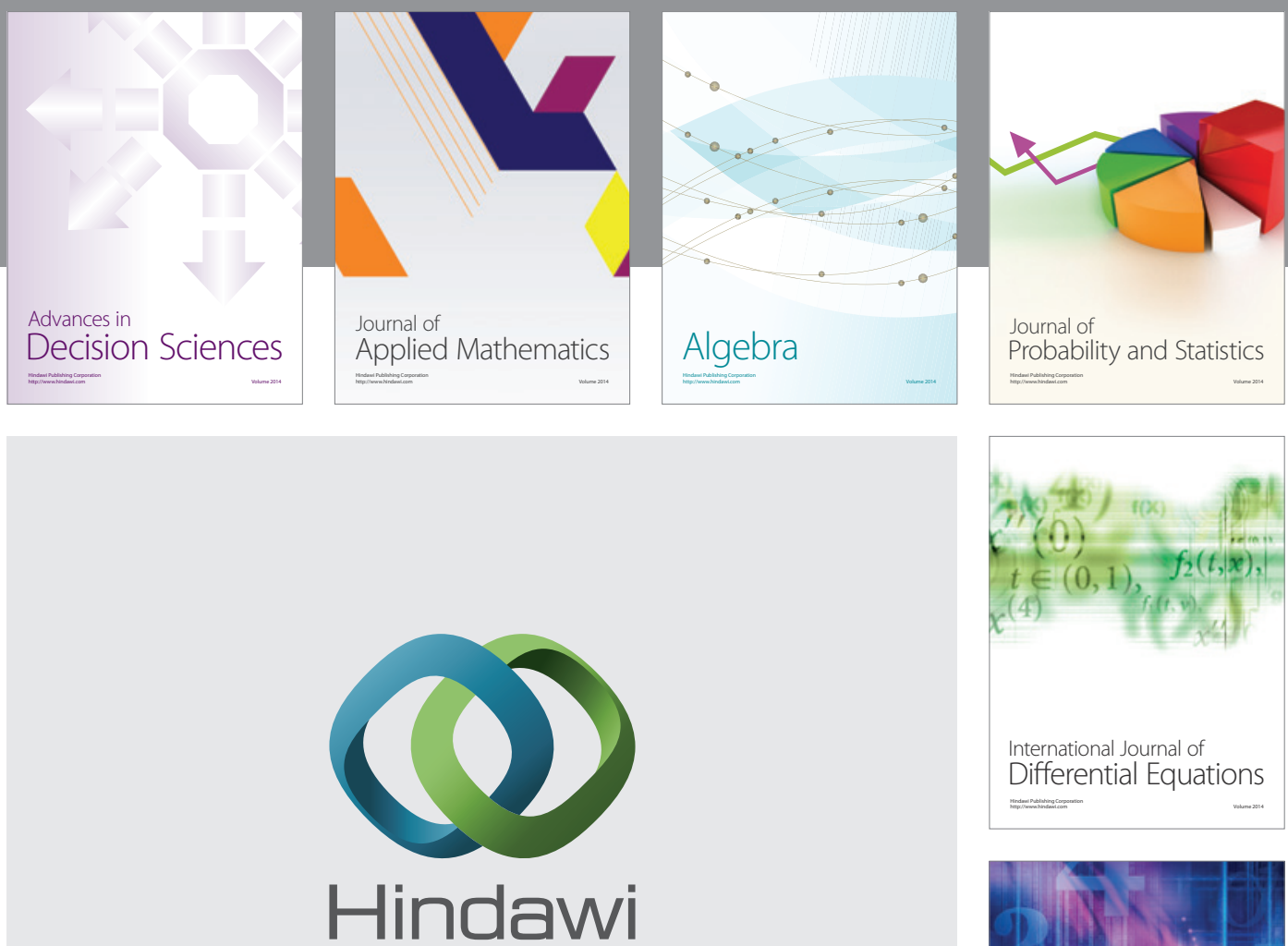

Submit your manuscripts at http://www.hindawi.com
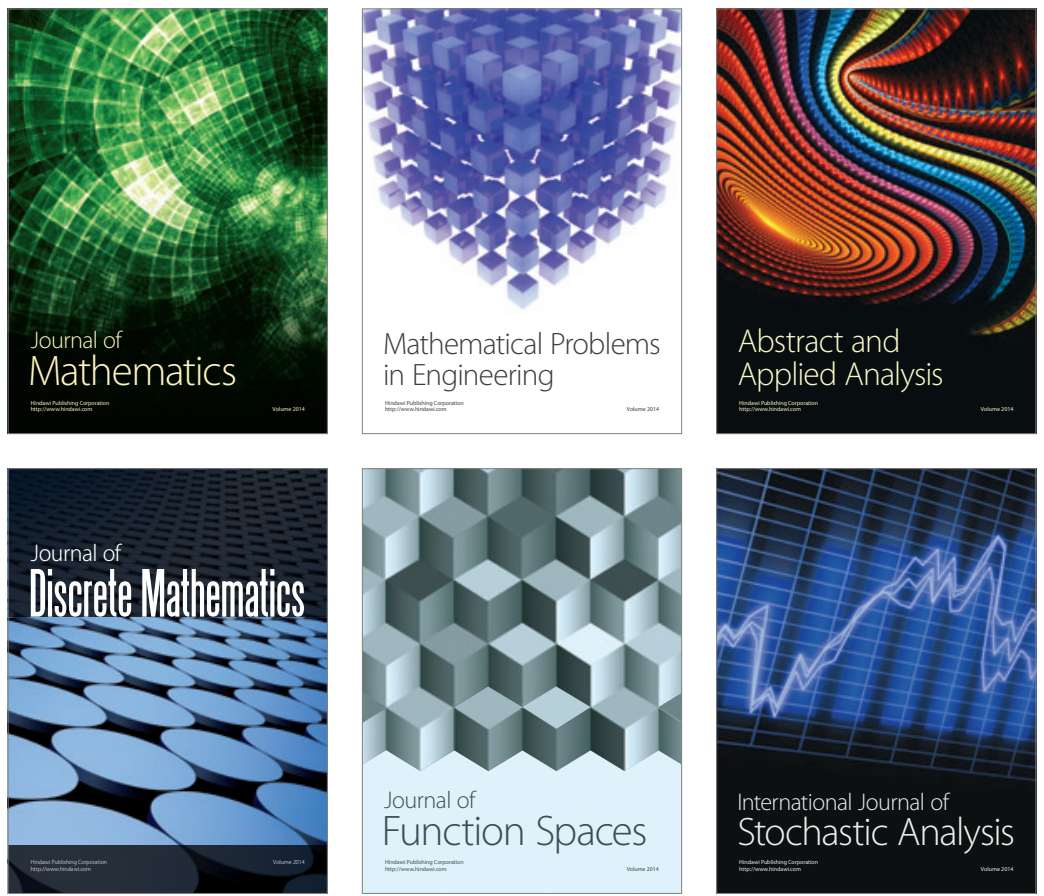

Journal of

Function Spaces

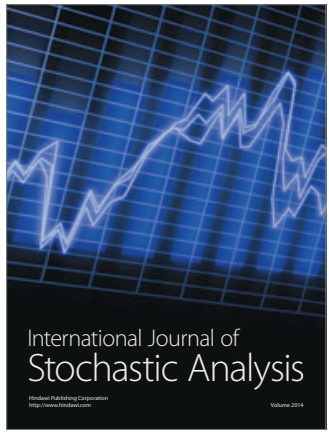

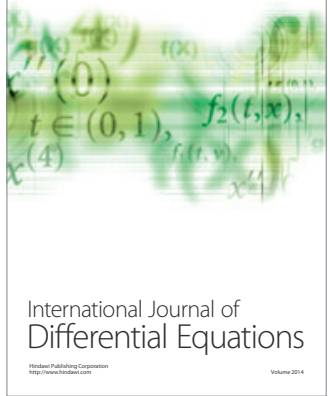
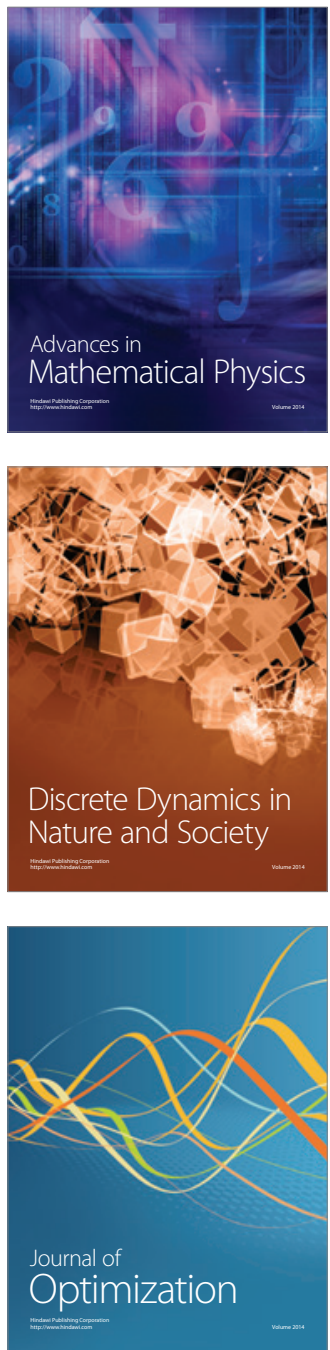